\title{
Florística e estrutura da vegetação arbustivo-arbórea das Areias Brancas do Parque Nacional Serra de Itabaiana/Sergipe, Brasil ${ }^{1}$
}

\author{
TÚLIO VINICIUS PAES DANTAS 2,4, JOSÉ ELVINO DO NASCIMENTO-JÚNIOR ${ }^{2}$, \\ ADAUTO DE SOUZA RIBEIRO ${ }^{3}$ e ANA PAULA DO NASCIMENTO PRATA ${ }^{2}$
}

(recebido: 11 de dezembro de 2008; aceito: 23 de setembro de 2010)

\begin{abstract}
Floristic and structure of the shrub and tree vegetation of Areias Brancas of Serra de Itabaiana National Park/ Sergipe, Brazil) The Serra de Itabaiana National Park is distinguished by its phytophysiognomic diversity, among which, a locally known as Areias Brancas (white sands), an open vegetation, ranging from herb-shrub to shrub-tree and classified by some authors as restinga or cerrado, depending on its size. Aiming to characterize and classify vegetational type were selected two sites in Areias Brancas of Serra de Itabaiana National Park and built a list following deposited material in the ASE Herbarium and additional sampling. To the phytosociological study it was used the wandering quarter method, sampling all individuals with trunk circumference $(>15 \mathrm{~cm})$ at breast height $(1.30 \mathrm{~m})$. It was verified, at the Areias Brancas, the occurrence of 193 species belonging to 145 genera and 60 families that are mostly represented by herb and shrub species. It is noticeable the high number of species found in Areias Brancas when compared with studies conducted throughout the National Park. There are significant floristic similarity between sampling sites, but structurally the two areas should be considered as different sucessional faces, due to variations in density, height and dominance. Even with plant species in common, the Areias Brancas of the National Park can not be classified as restinga or cerrado for not having common origins for these formations. The Areias Brancas should be considered as an ecological refuge, an azonal vegetation derived from the special conditions of substrate formation.
\end{abstract}

Key words - ecotone, refuge, succession

RESUMO - (Florística e estrutura da vegetação arbustivo-arbórea das Areias Brancas do Parque Nacional Serra de Itabaiana/ Sergipe, Brasil). O Parque Nacional Serra de Itabaiana destaca-se pela diversidade fitofisionômica, dentre estas, uma conhecida localmente por Areias Brancas, uma vegetação aberta que varia de arbustiva-herbácea a arbustiva-arbórea e classificada por alguns autores como restinga ou cerrado, a depender do porte. Com intuito caracterizar e classificar esta fitofisionomia foram selecionadas duas áreas amostrais de Areias Brancas no Parque Nacional Serra de Itabaiana e construída uma listagem de acordo com o material depositado no Herbário ASE e de coletas adicionais. Para o estudo fitossociológico foi utilizado o método dos quadrantes errantes, amostrando todos os indivíduos com circunferência do tronco $(>15 \mathrm{~cm})$ a altura do peito $(1,30 \mathrm{~m})$. Foi verificada nas Areias Brancas do Parque, a ocorrência de 193 espécies, distribuídas em 143 gêneros e 60 famílias, sendo, em sua maioria, representadas por espécies herbáceas e arbustivas. Destaca-se o alto número de espécies encontradas nas Areias Brancas quando comparado com estudos realizados em todo Parque Nacional. Há similaridade florística significativa entre as áreas amostradas, porém estruturalmente as duas áreas devem ser consideradas como diferentes fisionomias sucessionais pelas variações de densidade, altura e dominância. Mesmo possuindo espécies vegetais em comum, as Areias Brancas do Parque Nacional não podem ser classificadas como restinga ou cerrado por não possuir características de origem comuns a estas formações. As Areias Brancas devem ser consideradas como um refúgio ecológico, uma vegetação azonal derivada de condições especiais da formação do substrato.

Palavras-chave - ecótono, refúgio, sucessão

\section{Introdução}

O Estado de Sergipe é o menor estado da Federação brasileira, possuindo uma extensão aproximada de

1. Parte da dissertação de mestrado do primeiro autor, Programa de Pós-Graduação em Meio Ambiente e Desenvolvimento - Prodema, Universidade Federal de Sergipe, São Cristóvão, SE, Brasil.

2. Universidade Federal de Sergipe, Departamento de Biologia, Laboratório de Sistemática Vegetal. Rua Marechal Rondon, sn, 49100-000 São Cristóvão, SE, Brasil.

3. Universidade Federal de Sergipe, Departamento de Biologia, Laboratório de Biologia da Conservação. Rua Marechal Rondon, sn, 49100-000 São Cristóvão, SE, Brasil.

4. Autor para correspondência: tuliovpd@hotmail.com
$21.994 \mathrm{~km}^{2}$ (Embrapa 1975) apresenta uma pequena cobertura vegetal primitiva, possuindo algumas manchas de floresta costeira, matas de restingas e caatingas (Andrade \& Santos 1985).

O Parque Nacional Serra de Itabaiana está situado em uma área de ecótono entre Mata Atlântica e Caatinga, em uma zona de transição denominada de agreste, na qual coexistem espécies da flora de ambos os ecossistemas limítrofes. A posição geográfica do Parque Nacional lhe garante características especiais que favorecem a existência de diversos tipos vegetacionais a depender do solo e do relevo no qual se encontram. A devastação causada pelo homem resultou no isolamento dos 
fragmentos florestais da Serra em relação às florestas costeiras, e o próprio Parque Nacional já foi bastante devastado antes de sua implantação, resultando em um gradiente de vegetação em diversos estádios de regeneração, existindo poucas áreas de vegetação primária e contínua (Franco, 1983, Vicente et al. 2005).

As formações vegetais florestais do Parque Nacional são compostas por florestas secundárias localizadas nas encostas e acompanhando os riachos que recortam a Serra. As formações não florestais, por sua vez, foram classificadas em quatro diferentes habitats naturais: Areias Brancas, Vegetação Arbustiva, Áreas Úmidas e Áreas com Gramíneas e Ciperáceas (Vicente et al. 1997).

No habitat das Areias Brancas, uma comunidade arbustivo-herbácea esclerófila é distinta das vegetações de matas estacionais e de Caatinga hipoxerófila, características das regiões do agreste do Estado de Sergipe. A associação dos indivíduos em moitas é um atributo desta comunidade. As moitas podem ser formadas por indivíduos da mesma espécie, como Vellozia dasypus Seub. e Melocactus zehntneri Lutzelb. (Vilar et al. 2000) ou por diversas espécies em moitas mais complexas (Vicente et al. 2005).

Neste habitat, os solos são compostos inteiramente por areias brancas, comuns na face leste da Serra de Itabaiana e Serra Comprida, que conjuntamente com a Serra do Cajueiro, formam o complexo de Serras que compõem o Parque Nacional Serra de Itabaiana. Estes solos são formados por areias quartzosas distróficas, excessivamente drenados, moderado a extremamente ácidos e de baixa fertilidade natural (Embrapa 1975).

Observações e estudos nestas áreas têm descrito esta vegetação de uma maneira confusa em relação a sua caracterização. Ora esta vegetação era caracterizada como restinga (Governo de Sergipe 1976, 1978) quando possuía um porte baixo e pouco denso, ora como cerrado (Franco 1983, Gonçalves \& Orlandi, 1983, IBGE 2004) quando a vegetação era de maior porte havia ocorrência de espécies consideradas típicas do cerrado, como Curatella americana L. Seria possível que formações tão distintas quanto as restingas e os cerrados possam estar representadas em uma vegetação disjunta como nas Areias Brancas do Parque Nacional?

Interpretações mais recentes consideram que tais classificações não são condizentes, pois a região do Parque Nacional Serra de Itabaiana não possui características climáticas, de relevo ou origem comum a essas formações (Vicente et al. 1997, 2005).

Apesar destas observações rejeitarem a denominação da área como restingas ou cerrados, Vicente et al. (2005) ainda consideram a vegetação das Areias Brancas como formações diferentes, denominando-as de Areias Brancas e Vegetação Arbustiva para as vegetações arbustivas abertas e fechadas sobre o solo branco arenoso, respectivamente.

A vegetação aberta e fechada sobre solos de areias brancas podem ser consideradas como formações vegetacionais diferentes, mesmo que próximas e sob as mesmas condições abióticas?

Visando responder a esta questão e ampliar o conhecimento acerca desta formação vegetal, o presente estudo teve como objetivo realizar um levantamento florístico e comparar a riqueza de espécies e a estrutura fitossociológica do componente arbustivo-arbóreo de duas fitofisionomias de Areias Brancas no Parque Nacional Serra de Itabaiana, a fim de relacionar as semelhanças e diferenças entre estas vegetações que historicamente são consideradas distintas.

\section{Material e métodos}

Local de estudo - O Parque Nacional Serra de Itabaiana está inserido nos Municípios de Areia Branca, Itabaiana, Laranjeiras, Itaporanga d'Ajuda e Campo do Brito, no Estado de Sergipe, Nordeste do Brasil. Está localizado entre as latitudes e longitudes aproximadas de $10^{\circ} 40^{\prime} \mathrm{S}$ e $37^{\circ} 25^{\prime} \mathrm{O}$, apresentando cerca de $670 \mathrm{~m}$ de altitude máxima e distante $45 \mathrm{~km}$ de Aracaju. O clima da região é, segundo a classificação de Köeppen (Köppen \& Geiger 1928), As' - clima tropical com verão seco e moderado excedente hídrico no inverno com índice de Thorntwaite (Im) entre - 1,3 e 8,8 (Vicente 1999).

Foram selecionadas duas áreas amostrais, uma localizada entre os riachos Coqueiro e Água Fria $\left(10^{\circ} 45^{\prime} 32^{\prime \prime} \mathrm{S}\right.$ e $37^{\circ} 20^{\prime} 59^{\prime \prime} \mathrm{O}$ ), considerada como vegetação de Areias Brancas abertas, e outra adjacente a sede do Ibama, entre os

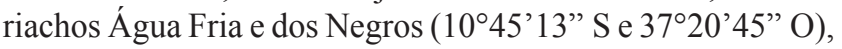
denominada Areias Brancas fechadas. As duas áreas estão isoladas entre si por uma vegetação de mata mesófila ao longo do Riacho Água Fria.

Composição florística - Para construção da lista de espécies, foram realizadas consultas à coleção do Herbário ASE (acrônimo, segundo Holmgren \& Holmgren 1998), da Universidade Federal de Sergipe, e anotadas todas as espécies com ocorrência para as Areias Brancas da Serra de Itabaiana. Foram realizadas coletas adicionais nas Areias Brancas no período de novembro de 2007 a setembro de 2008, seguindo os procedimentos recomendados por Fidalgo \& Bononi (1984). As famílias botânicas estão apresentadas em ordem alfabética e citadas de acordo com o APG III (2009).

Estrutura arbustivo-arbórea - Para o estudo fitossociológico, utilizou-se o método dos quadrantes errantes (Catana \& Anthony 1963, Brower et al. 1997), com o cálculo da densidade corrigida por Brower et al. (1997). Foram amostrados todos os indivíduos que apresentavam circunferência do tronco a 
1,30 m do solo (CAP), maior ou igual a $15 \mathrm{~cm}$, exceto para os indivíduos que perfilharam acima do solo e abaixo da altura do peito $(1,30 \mathrm{~m})$. Nestes casos foi adotado o seguinte critério: o indivíduo foi incluído quando pelo menos uma das ramificações obedecia ao critério de inclusão $(\mathrm{CAP} \geq 15 \mathrm{~cm})$, sendo então anotado o CAP de todas as ramificações para o cálculo da área basal. Foi escolhido um ponto aleatório em mapa do local, para cada área, onde se começaram as amostragens. A partir destes pontos, o primeiro transecto foi traçado no sentido Sul-Norte, e para os transectos seguintes, distanciou-se em $25 \mathrm{~m}$ a Leste do anterior. $\mathrm{Na}$ área de Areias Brancas abertas foram amostrados dez transectos e nas Areias Brancas fechadas oito em janeiro de 2007. A distribuição espacial dos indivíduos amostrados no levantamento fitossociologico foi determinada pelo (IAP) Índice de Agregação de Payandeh (Payandeh 1970), medido através da razão entre variância das distâncias entre as plantas e média destas distâncias: valores menores que 1,0 indicam distribuição regular; entre 1,0 e 1,5 indicam distribuição aleatória e valores maiores que 1,5 distribuição agregada.

Similaridade florística e estrutural entre as fisionomias fechada e aberta - Para verificar a similaridade florística entre as duas áreas amostradas no levantamento fitossociológico usamos o índice de similaridade de Sorensen (Brower et al. 1997). Para verificar se as fisionomias possuem vegetações estruturalmente semelhantes, partimos da hipótese $\left(\mathrm{H}_{0}\right)$ de que estas fazem parte da mesma metapopulação. Para tanto, utilizamos o teste " $\mathrm{t}$ " para variáveis independentes no intuito de comparar as estruturas etárias (diâmetro de caule) e de altura das duas áreas. Para todas as análises foram utilizados os níveis de significância de 0,05 . Foi calculada a Diversidade pelo índice de Shannon-Weinner $\left(H^{\prime}\right)$ em base $e$, conforme Brower et al. (1997) e a Equabilidade (Pielou 1975).

\section{Resultados e discussão}

Composição florística - Foi verificada a ocorrência de 193 espécies no habitat de Areias Brancas do Parque Nacional Serra de Itabaiana, distribuídas em 145 gêneros e 60 famílias (tabela 1). O número de espécies encontradas nas Areias Brancas do Parque Nacional Serra de Itabaiana foi bastante expressivo, quando comparados à listagem florística elaborada por Vicente et al. (2005), na qual são referidas 215 espécies vegetais para todo o Parque Nacional Serra de Itabaiana.

As famílias com maior riqueza específica para a área foram Fabaceae (24), Rubiaceae (14), Asteraceae (11), Poaceae (9), Apocynaceae e Cyperaceae (8). Do total das espécies citadas 30 possuem porte arbóreo, 46 arbustivo, 23 são subarbustos, 71 são herbáceas, 15 são trepadeiras, quatro hemiparasitas, duas epífita, uma hemiepifita, e uma parasita. Encontrando-se uma maior abundância de espécies arbustivo-herbáceas para a vegetação das Areias Brancas.

O estrato arbóreo é ralo e disperso. Os elementos mais freqüentes com este tipo de hábito são Acosmium bijugum, Kielmeyera rugosa e Agarista revoluta. Muitas das árvores encontradas nas áreas de Areias Brancas são

Tabela 1. Listagem florística das vegetações das Areias Brancas do Parque Nacional Serra de Itabaiana, Sergipe, Brasil.

Table 1. Floristic checklist of National Park Serra de Itabaiana's Areias Brancas vegetation, Sergipe State, Brazil.

\begin{tabular}{|c|c|c|}
\hline Famílias/Espécies & Hábito & Coletor/№ \\
\hline \multicolumn{3}{|l|}{ ACANTHACEAE } \\
\hline Geissomeria sp. & Arbusto & A. Silva (ASE 5376) \\
\hline Ruellia bahiensis (Nees) Morong & Erva & G.Viana 1325 \\
\hline \multicolumn{3}{|l|}{ AMARANTHACEAE } \\
\hline Gomphrena demissa Mart. & Erva & T.V.P. Dantas 55 \\
\hline Amaranthaceae sp. & Erva & T.V.P. Dantas 13 \\
\hline \multicolumn{3}{|l|}{ ANACARDIACEAE } \\
\hline Anacardium occidentale L. & Árvore & \\
\hline Thyrsodium sp. & Árvore & A.C. Barreto (ASE 561) \\
\hline \multicolumn{3}{|l|}{ APOCYNACEAE } \\
\hline Ditassa crassifolia Decne. & Trepadeira & T.V.P. Dantas 53 \\
\hline Mandevilla hirsuta (Rich.) K. Schum. & Trepadeira & T.V.P. Dantas 70 \\
\hline Mandevilla moricandiana (A. DC.) Woodson & Trepadeira & T.V.P. Dantas 01 \\
\hline Mandevilla sp. 1 & Trepadeira & E. Carneiro 281 \\
\hline Hancornia speciosa B. A. Gomes & Árvore & M. Fonseca (ASE 247) \\
\hline Himatanthus bracteatus (A. DC.) Woodson & Árvore & A. Silva (ASE 5374) \\
\hline Himatanthus obovatus (Vahl) Woodson & Árvore & \\
\hline
\end{tabular}


continuação

\begin{tabular}{|c|c|c|}
\hline Famílias/Espécies & Hábito & Coletor/№ \\
\hline \multicolumn{3}{|l|}{ ARACEAE } \\
\hline Anthurium affine Schott & Erva & \\
\hline Anthurium bellum Schott & Erva & J.E. Nascimento Jr (ASE 7567) \\
\hline Caladium bicolor (Aiton) Vent. & Erva & G.Viana 1761 \\
\hline Philodendron acutatum Schott & Hemiepífita & \\
\hline \multicolumn{3}{|l|}{ ARALIACEAE } \\
\hline $\begin{array}{l}\text { Schefflera vinosa (Cham. \& Schltdl.) Frodin \& Fiaschi } \\
\text { ARECACEAE }\end{array}$ & ARECACEAE & E. Carneiro 364 \\
\hline Allagoptera campestris (Mart.) Kuntze & Arbusto & M. Fonseca (ASE 152) \\
\hline Attalea funifera Mart. & Arbusto & \\
\hline Bactris sp. & Arbusto & A.C. Barreto (ASE 564) \\
\hline Syagrus schizophylla (Mart.) Glassman & Árvore & \\
\hline \multicolumn{3}{|l|}{ ARISTOLOCHIACEAE } \\
\hline \multirow{2}{*}{\multicolumn{3}{|c|}{ ASTERACEAE }} \\
\hline & & \\
\hline Acritopappus confertus (Gardner) R. M. King \& H. Rob. & Arbusto & T.V.P. Dantas 18 \\
\hline Aspilia martii Baker & Erva & T.V.P. Dantas 25 \\
\hline Calea $\mathrm{sp}$. & Erva & G. Viana 1255 \\
\hline Elephantopus hirtiflorus DC. & Erva & T.V.P. Dantas 79 \\
\hline Emilia sonchifolia $(\mathrm{L}$.$) DC.$ & & T.V.P. Dantas 61 \\
\hline Erechtites hieraciifolius (L.) Raf. ex DC. & Erva & T.V.P. Dantas 58 \\
\hline Eremanthus reflexo-auriculatus G. M. Barroso & Erva & M. Fonseca (ASE 249) \\
\hline Ichthyothere terminalis (Spreng.) S. F. Blake & Erva & E. Gomes 11 \\
\hline Lepidaploa chalybaea (Mart. ex DC.) H. Rob. & Subarbusto & T.V.P. Dantas 76 \\
\hline Mikania nodulosa Sch. Bip. ex Baker & Trepadeira & G.Viana 411 \\
\hline Tilesia baccata (L.) Pruski & Arbusto & T.V.P. Dantas 59 \\
\hline \multicolumn{3}{|l|}{ BIGNONIACEAE } \\
\hline Jacaranda obovata Cham. & Árvore & T.V.P. Dantas 37 \\
\hline \multicolumn{3}{|l|}{ BORAGINACEAE } \\
\hline Varronia curassavica Jacq. & Arbusto & T.V.P. Dantas 24 \\
\hline \multicolumn{3}{|l|}{ BROMELIACEAE } \\
\hline Aechmea mertensii (G. Mey.) Schult. \& Schult. f. & Epífita & C.L. Souza 19 \\
\hline Hohenbergia catingae Ule & Erva & M. Landim 716 \\
\hline Tillandsia recurvata $(\mathrm{L}.) \mathrm{L}$. & Epífita & \\
\hline Vriesea procera (Mart. ex Schult. \& Schult.f.) Wittm. & Erva & J. E Nascimento-Júnior 538 \\
\hline \multicolumn{3}{|l|}{ BURSERACEAE } \\
\hline Protium heptaphyllum (Aubl.) Marchand & Árvore & T.V.P. Dantas 33 \\
\hline \multicolumn{3}{|l|}{ CACTACEAE } \\
\hline Melocactus zehntneri (Britt. \& Rose) Luetzelburg & Erva & T.V.P. Dantas 92 \\
\hline \multicolumn{3}{|l|}{ CELASTRACEAE } \\
\hline Maytenus opaca Reissek & Arbusto & M. Fonseca 432 \\
\hline \multicolumn{3}{|l|}{ CHRYSOBALANACEAE } \\
\hline Hirtella racemosa Lam. & Arbusto & E. Carneiro 352 \\
\hline \multicolumn{3}{|l|}{ CLUSIACEAE } \\
\hline Clusia nemorosa G. F. M. Meyer & Árvore & Cynara (ASE 10862) \\
\hline Clusia sp. & Árvore & M. Landim 899 \\
\hline Kielmeyera argentea Choisy & Arbusto & G.Viana 1247 \\
\hline Kielmeyera rugosa Choisy & Árvore & T.V.P. Dantas 35 \\
\hline \multicolumn{3}{|l|}{ COMMELINACEAE } \\
\hline Murdannia nudiflora (L.) Brenan & Erva & M.C. Santana 69 \\
\hline Commelina sp. & Erva & G.Viana 1251 \\
\hline
\end{tabular}


continuação

\begin{tabular}{|c|c|c|}
\hline Famílias/Espécies & Hábito & Coletor/№ \\
\hline \multicolumn{3}{|l|}{ CONVOLVULACEAE } \\
\hline Evolvulus pterocaulon Moric. & Erva & C.L. Souza 11 \\
\hline Evolvulus phyllanthoides Moric. & Erva & T.V.P. Dantas 21 \\
\hline Jacquemontia montana (Moric.) Meisn. & Trepadeira & E. Carneiro 356 \\
\hline Jacquemontia bahiensis O’Donell & Trepadeira & T.V.P. Dantas 69 \\
\hline \multicolumn{3}{|l|}{ CYPERACEAE } \\
\hline Bulbostylis capillaris (L.) C. B. Clarke & Erva & T.V.P. Dantas 47 \\
\hline Bulbostylis conifera (Kunth) C. B. Clarke & Erva & T.V.P. Dantas 48 \\
\hline Bulbostylis junciformis (Kunth) C. B. Clarke & Erva & T.V.P. Dantas 08 \\
\hline Cyperus hermaphroditus (Jacq.) Standl. & Erva & T.V.P. Dantas 31 \\
\hline Lagenocarpus rigidus Nees. & Erva & \\
\hline Lagenocarpus verticillatus (Spreng.) T. Koyama \& Maguire & Erva & T.V.P. Dantas 28 \\
\hline Rhynchospora barbata (Vahl) Kunth & Erva & T.V.P. Dantas 82 \\
\hline Rhynchospora nervosa (Vahl) Boeck. & Erva & A.C. Barreto (ASE 273) \\
\hline \multicolumn{3}{|l|}{ DILLENIACEAE } \\
\hline Curatella americana $\mathrm{L}$. & Árvore & E. Carneiro 278 \\
\hline Davilla flexuosa A. St.-Hil. & Arbusto & M. Fonseca 440 \\
\hline Tetracera breyniana Schltdl. & Arbusto & A.C. Barreto (ASE 560) \\
\hline \multicolumn{3}{|l|}{ DIOSCOREACEAE } \\
\hline Dioscorea ovata Vell. & Trepadeira & Givane (ASE 325) \\
\hline Dioscorea sp. & Trepadeira & T.V.P. Dantas 39 \\
\hline \multicolumn{3}{|l|}{ ERICACEAE } \\
\hline Gaylussacia brasiliensis (Spreng.) Meisn. & Arbusto & M. Fonseca (ASE 154) \\
\hline Agarista revoluta (Spreng.) Hook. f. ex Nied. & Árvore & T.V.P. Dantas 45 \\
\hline \multicolumn{3}{|l|}{ ERIOCAULACEAE } \\
\hline Paepalanthus bifidus (Schrader) Kunth & Erva & A.C. Barreto (ASE 574) \\
\hline Paepalanthus myocephalus Mart. & Erva & T.V.P. Dantas 26 \\
\hline Syngonanthus nitens (Bong.) Ruhland & Erva & G. Viana 5 \\
\hline Syngonanthus imbricatus (Koern.) Ruhland & Erva & M. Fonseca 441 \\
\hline Eriocaulaceae sp. & Erva & T.V.P. Dantas 40 \\
\hline \multicolumn{3}{|l|}{ EUPHORBIACEAE } \\
\hline Croton pedicellatus Kunth & Arbusto & T.V.P. Dantas 72 \\
\hline Euphorbia thymifolia $\mathrm{L}$. & Subarbusto & T.V.P. Dantas 06 \\
\hline Microstachys corniculata (Vahl) Griseb. & Trepadeira & T.V.P. Dantas 23 \\
\hline Microstachys sp. & Subarbusto & T.V.P. Dantas 51 \\
\hline Pera glabrata (Schott) Poepp. ex Baill. & Árvore & T.V.P. Dantas 85 \\
\hline Euphorbiaceae 1 & Arbusto & \\
\hline \multicolumn{3}{|l|}{ FABACEAE } \\
\hline Acosmium bijugum (Vogel) Yakovlev & Árvore & M. Landim 506 \\
\hline Andira laurifolia Benth. & Arbusto & A.C. Barreto (ASE 382) \\
\hline Bauhinia acuruana Moric. & Árvore & T.V.P. Dantas 36 \\
\hline Bowdichia virgilioides Kunth & Árvore & G. Viana 340 \\
\hline Calopogonium caeruleum (Benth.) C. Wright & Trepadeira & T.V.P. Dantas 75 \\
\hline Centrosema brasilianum (L.) Benth. & Trepadeira & T.V.P. Dantas 62 \\
\hline Chamaecrista cytisoides (DC. ex Collad.) H. S. Irwin \& Barneby & Árvore & T.V.P. Dantas 03 \\
\hline Chamaecrista desvauxii (Collad.) Killip & Subarbusto & G. Viana 63 \\
\hline Chamaecrista flexuosa (L.) Greene & Subarbusto & T.V.P. Dantas 77 \\
\hline Chamaecrista hispidula (Vahl) H. S. Irwin \& Barneby & Erva & G. Viana 1965 \\
\hline Chamaecrista ramosa (Vogel) H. S. Irwin \& Barneby & Subarbusto & T.V.P. Dantas 16 \\
\hline Clitoria laurifolia Poir. & Subarbusto & G. Viana 1811 \\
\hline Clitoria guianensis (Aubl.) Benth. & Erva & M. A. Figueiredo 22 \\
\hline
\end{tabular}


continuação

\begin{tabular}{|c|c|c|}
\hline Famílias/Espécies & Hábito & Coletor/№ \\
\hline Crotalaria pallida Aiton & Subarbusto & C. Almeida 15 \\
\hline Crotalaria sp. & Subarbusto & G. Viana 1262 \\
\hline Desmodium barbatum (L.) Benth. & Erva & T.V.P. Dantas 12 \\
\hline Diptychandra aurantiaca Tul. & Árvore & M.C. Santana 77 \\
\hline Periandra mediterranea (Vell.) Taub. & Arbusto & \\
\hline Senna macranthera (DC. ex Collad.) H. S. Irwin \& Barneby & Arbusto & M.C. Santana 71 \\
\hline Stryphnodendron pulcherrimum (Willd.) Hochr. & Árvore & M.C. Santana 53 \\
\hline Stylosanthes guianensis (Aubl.) Sw. & Subarbusto & T.V.P. Dantas 83 \\
\hline Stylosanthes scabra Vogel & Subarbusto & M. Landum 1062 \\
\hline Stylosanthes viscosa (L.) Sw. & Subarbusto & T.V.P. Dantas 86 \\
\hline \multicolumn{3}{|l|}{ GENTIANACEAE } \\
\hline Chelonanthus purpurascens (Aubl.) Struwe \& V. A. Albert & Erva & G. Viana 1270 \\
\hline \multicolumn{3}{|l|}{ HUMIRIACEAE } \\
\hline Humiria balsamifera Aubl. & Arbusto & M. Fonseca 435 \\
\hline \multicolumn{3}{|l|}{ HYPERICACEAE } \\
\hline Vismia guianensis (Aubl.) Pers. & Arbusto & G. Viana 435 \\
\hline \multicolumn{3}{|l|}{ KRAMERIACEAE } \\
\hline Krameria grandiflora A. St.-Hil. & Hemiparasita & T.V.P. Dantas 17 \\
\hline \multicolumn{3}{|l|}{ LAMIACEAE } \\
\hline Hyptis fruticosa Salzm. ex Benth. & Arbusto & A.C. Barreto (ASE 374) \\
\hline Marsypianthes chamaedrys (Vahl) Kuntze & Erva & T.V.P. Dantas 19 \\
\hline \multicolumn{3}{|l|}{ LAURACEAE } \\
\hline Cassytha filiformis L. & Erva parasita & A.C. Barreto (ASE 580) \\
\hline Ocotea duckei Vattimo & Arbusto & A.C. Barreto (ASE 526) \\
\hline Ocotea gardneri (Meisn.) Mez & Árvore & A.C. Barreto (ASE 7622) \\
\hline \multicolumn{3}{|l|}{ LORANTHACEAE } \\
\hline Psittacanthus bicalyculatus Mart. & Hemiparasita & T.V.P. Dantas 34 \\
\hline Psittacanthus dichrous (Mart.) Mart. & Hemiparasita & M. Landim 782 \\
\hline \multicolumn{3}{|l|}{ LYTRACEAE } \\
\hline Cuphea flava Spreng. & Subarbusto & T.V.P. Dantas 57 \\
\hline Cuphea pulchra Moric. & Subarbusto & G. Viana 04 \\
\hline Lafoensia sp. 1 & Árvore & G. Viana 1843 \\
\hline Lafoensia sp. 2 & Árvore & T.V.P. Dantas 87 \\
\hline \multicolumn{3}{|l|}{ MALPIGHIACEAE } \\
\hline Byrsonima sp. & Arbusto & G. Viana 834 \\
\hline Byrsonima sericea DC. & Árvore & G. Viana 1245 \\
\hline Stigmaphyllon paralias A. Juss. & Subarbusto & M.A. Figueiredo 23 \\
\hline \multicolumn{3}{|l|}{ MALVACEAE } \\
\hline Sida cordifolia $\mathrm{L}$. & Erva & G. Viana 1252 \\
\hline Sida ciliaris L. & Subarbusto & G. Viana 1263 \\
\hline \multicolumn{3}{|l|}{ MARANTACEAE } \\
\hline Maranta sp. & Erva & G. Viana 416 \\
\hline \multicolumn{3}{|l|}{ MARCGRAVIACEAE } \\
\hline Schwartzia brasiliensis (Choisy) Bedell ex Gir.-Cañas & Arbusto & G. Viana 1657 \\
\hline \multicolumn{3}{|l|}{ MELASTOMATACEAE } \\
\hline Aciotis sp. & Erva & T.V.P. Dantas 52 \\
\hline Cambessedesia tenuis Markgr. & Arbusto & M. Fonseca 443 \\
\hline Clidemia capitellata (Bonpl.) D. Don & Arbusto & G. Viana 1814 \\
\hline Miconia albicans (Sw.) Triana & Arbusto & \\
\hline Miconia ciliata (Rich.) DC. & Arbusto & \\
\hline Tibouchina sp. & Arbusto & E. Carneiro 276 \\
\hline
\end{tabular}


continuação

\begin{tabular}{|c|c|c|}
\hline Famílias/Espécies & Hábito & Coletor/№ \\
\hline \multicolumn{3}{|l|}{ MYRTACEAE } \\
\hline Eugenia punicifolia (Kunth) DC. & Arbusto & C.L. Souza 17 \\
\hline Myrcia splendens (Sw.) DC. & Arbusto & Cruz (ASE 11470) \\
\hline Myrcia guianensis (Aubl.) DC. & Arbusto & G. Viana 1653 \\
\hline Myrcia lundiana Kiaersk. & Arbusto & T.V.P. Dantas 32 \\
\hline Myrciaria floribunda (H. West ex Willd.) O. Berg & Arbusto & E.Carneiro 362 \\
\hline \multicolumn{3}{|l|}{ NYCTAGINACEAE } \\
\hline Guapira opposita (Vell.) Reitz & Árvore & M. C. Santana 51 \\
\hline Pisonia cordifolia Mart. & Arbusto & M. Landim 780 \\
\hline \multicolumn{3}{|l|}{ OCHNACEAE } \\
\hline Ouratea cuspidata Tiegh. & Árvore & G. Viana (ASE 4784) \\
\hline Sauvagesia erecta $\mathrm{L}$. & Arbusto & C. Almeida 17 \\
\hline \multicolumn{3}{|l|}{ ORCHIDACEAE } \\
\hline Cyrtopodium glutiniferum Raddi & Erva & G. Viana 234 \\
\hline Encyclia dichroma (Lindl.) Schltr. & Erva & T.V.P. Dantas 43 \\
\hline Epistephium lucidum Cogn. & Erva & G. Viana 528 \\
\hline Habenaria petalodes Lindl. & Erva & T.V.P. Dantas 66 \\
\hline Sobralia liliastrum Lindl. & Erva & G. Viana 821 \\
\hline Orchidaceae sp. & Erva & E. Carneiro 277 \\
\hline \multicolumn{3}{|l|}{ OROBANCHACEAE } \\
\hline Buchnera longifolia Kunth & Erva & M.C.Santana 76 \\
\hline \multicolumn{3}{|l|}{ OXALIDACEAE } \\
\hline Oxalis divaricata Mart. ex Zucc. & Erva & G. Viana 1836 \\
\hline \multicolumn{3}{|l|}{ PHYTOLACCACEAE } \\
\hline Phytolacca dioica $\mathrm{L}$. & Arbusto & G. Viana 1236 \\
\hline \multicolumn{3}{|l|}{ PLANTAGINACEAE } \\
\hline Angelonia cornigera Hook. & Erva & T.V.P. Dantas 71 \\
\hline Scoparia dulcis L. & Erva & G. Viana 409 \\
\hline Stemodia foliosa Benth. & Subarbusto & E. Gomes 21 \\
\hline \multicolumn{3}{|l|}{ PASSIFLORACEAE } \\
\hline Piriqueta duarteana (Cambess.) Urb. & Erva & T.V.P. Dantas 15 \\
\hline Turnera subulata $\mathrm{Sm}$. & Erva & M. Fonseca 10 \\
\hline Turnera chamaedrifolia Cambess. & Erva & C. Almeida 14 \\
\hline \multicolumn{3}{|l|}{ POACEAE } \\
\hline Andropogon bicornis L. & Erva & T.V.P. Dantas 27 \\
\hline Aristida setifolia Kunth & Erva & T.V.P. Dantas 29 \\
\hline Axonopus aureus P. Beauv. & Erva & T.V.P. Dantas 30 \\
\hline Echinolaena inflexa (Poir.) Chase & Erva & M. Fonseca (ASE 220) \\
\hline Eragrostis articulata (Schrank) Nees & Erva & T.V.P. Dantas 20 \\
\hline Eragrostis rufescens Schrad. ex Schult. & Erva & T.V.P. Dantas 60 \\
\hline Panicum trichoides $\mathrm{Sw}$. & Erva & T.V.P. Dantas 56 \\
\hline Paspalum arenarium Schrad. & Erva & T.V.P. Dantas 63 \\
\hline Trachypogon spicatus (L. f.) Kuntze & Erva & T.V.P. Dantas 81 \\
\hline \multicolumn{3}{|l|}{ POLYGALACEAE } \\
\hline Polygala glochidiata Kunth & Erva & T.V.P. Dantas 50 \\
\hline Polygala trichosperma L. & Erva & T.V.P. Dantas 10 \\
\hline \multicolumn{3}{|l|}{ POLYGONACEAE } \\
\hline Coccoloba laevis Casar. & Arbusto & A.C. Barreto (ASE 7375) \\
\hline Coccoloba rosea Meisn. & Arbusto & T.V.P. Dantas 44 \\
\hline \multicolumn{3}{|l|}{ RUBIACEAE } \\
\hline Alibertia sp. & Árvore & T.V.P. Dantas 89 \\
\hline
\end{tabular}


continuação

\begin{tabular}{|c|c|c|}
\hline Famílias/Espécies & Hábito & Coletor/№ \\
\hline Borreria capitata (Ruiz \& Pav.) DC. & Erva & T.V.P. Dantas 49 \\
\hline Borreria eryngioides Cham. \& Schltdl. & Subarbusto & T.V.P. Dantas 54 \\
\hline Borreria humifusa Mart. & Subarbusto & T.V.P. Dantas 22 \\
\hline Borreria verticillata (L.) G. Mey. & Subarbusto & T.V.P. Dantas 11 \\
\hline Declieuxia marioides Mart. \& Zucc. ex Schult. \& Schult. & Subarbusto & G. Viana 1302 \\
\hline Diodella radula (Willd. ex Roem. \& Schult.) Delprete & Erva & G. Viana 1805 \\
\hline Guettarda angelica Mart. ex Müll. Arg. & Arbusto & \\
\hline Guettarda platypoda DC. & Arbusto & T.V.P. Dantas 41 \\
\hline Leptoscela ruelloides Hook. f. & Erva & G. Viana 1742 \\
\hline Oldenlandia filicaulis K. Schum. & Erva & T.V.P. Dantas 73 \\
\hline Richardia grandiflora (Cham. \& Schult.) Schult \&Schult & Erva & T.V.P. Dantas 78 \\
\hline Salzmannia nitida DC. & Arbusto & T.V.P. Dantas 88 \\
\hline Rubiaceae sp. 1 & Subarbusto & T.V.P. Dantas 65 \\
\hline Rubiaceae sp. 2 & Subarbusto & T.V.P. Dantas 22 \\
\hline \multicolumn{3}{|l|}{ RUTACEAE } \\
\hline Esenbeckia grandiflora Mart. & Arbusto & T.V.P. Dantas 46 \\
\hline \multicolumn{3}{|l|}{ SANTALACEAE } \\
\hline Phoradendron affine (Pohl ex DC.) Engl. \& K. Krause & Hemiparasita & T.V.P. Dantas 42 \\
\hline \multicolumn{3}{|l|}{ SAPINDACEAE } \\
\hline Serjania salzmanniana Schltr. & Trepadeira & T.V.P. Dantas 84 \\
\hline \multicolumn{3}{|l|}{ SAPOTACEAE } \\
\hline Manilkara salzmannii (A. DC.) H. J. Lam & Árvore & T.V.P. Dantas 38 \\
\hline \multicolumn{3}{|l|}{ SMILACACEAE } \\
\hline Smilax sp. & Trepadeira & T.V.P. Dantas 05 \\
\hline \multicolumn{3}{|l|}{ VELLOZIACEAE } \\
\hline Vellozia dasypus Seub. & Erva & T.V.P. Dantas 04 \\
\hline \multicolumn{3}{|l|}{ VERBENACEAE } \\
\hline Lantana fucata Lindl & Arbusto & T.V.P. Dantas 09 \\
\hline Lantana radula $\mathrm{Sw}$. & Arbusto & T.V.P. Dantas 14 \\
\hline Lantana canescens Kunth & Arbusto & G. Viana 1239 \\
\hline Lippia sp. & Erva & G. Viana 1246 \\
\hline \multicolumn{3}{|l|}{ VOCHYSIACEAE } \\
\hline Vochysia lucida Klotzsch ex M. R. Schomb. & Árvore & G. Viana 668 \\
\hline
\end{tabular}

baixas (menores que seis metros) e apresentam grande incidência de caules cespitosos, confundindo-se com os arbustos.

Acosmium bijugum e Kielmeyera rugosa são espécies com distribuição restrita nos Estados da Bahia e Sergipe, em regiões de Restinga (Brito et al. 1993, Saddi 1994), enquanto Agarista revoluta, com distribuição mais ampla, já foi descrita em restingas da Bahia (Brito et al. 1993) e Rio de Janeiro (Rizzini 1979).

Muitos arbustos ocorrem agregados, formando moitas, onde há associações de dois ou mais arbustos na formação destas. Coccoloba laevis, Guettarda platypoda, Coccoloba rosea, Humiria balsamifera, Davilla flexuosa e Varronia curassavica são exemplos destes arbustos que habitam as Areias Brancas do Parque Nacional Serra de Itabaiana. Coccoloba laevis ocorre nas restingas de
Pernambuco (Zickel et al. 2007), da Bahia (Queiroz 2007) e na mata atlântica da Paraíba (Pereira \& Alves 2007). Guettarda platypoda distribui-se por todos os Estados do Nordeste do Brasil e nas restingas da Paraíba, Pernambuco e Bahia (Pereira \& Barbosa 2004, Zickel et al. 2007, Viana et al. 2006). Davilla flexuosa é uma espécies comuns nas restingas brasileiras e em áreas de solo arenoso em geral (David et al. 1996). Varronia curassavica foi citada por Rodal \& Nascimento (2002) na caatinga de Pernambuco, habitando a floresta de altitude e as baixadas de vegetação xerófita e Humiria balsamifera é uma espécie neotropical que ocorrem em toda América do Sul, em habitats florestados ou abertos (Cuatrecasas 1961).

As espécies herbáceas são mais abundantes nas Areias Brancas em relação a outros hábitos, porém 
não formam um continuum, ocorrendo dispersas entre os arbustos ou no solo desnudo. Vilar et al. (2000) descrevem a distribuição espacial de Vellozia dasypus e Melocactus zehntneri nas Areias Brancas do Parque, encontrando um padrão agregado de distribuição, motivado pelo modo de dispersão e pela reprodução vegetativa de seus órgãos subterrâneos.

Os subarbustos são encontrados prioritariamente isolados em regiões de solo desnudo, sendo em sua maioria espécies micrófilas esclerófilas da família Fabaceae. As mais abundantes são Cuphea pulchra, com ocorrência nas caatingas da Bahia e Minas Gerais, Cambessedesia tenuis, nas restingas da Bahia (Pilger 1927); Stylosanthes gracilis nas restingas de Pernambuco (Zickel et al. 2007); Borreria verticillata e Crotalaria mucronata, que são espécies cosmopolitas que ocorrem em toda América Central e do Sul (Boghossian et al. 2007).

Nas Areias Brancas do Parque foram encontradas quatro espécies de hemi-parasitas. Dentre elas se destaca Psittacanthus dichrous por sua abundância, sendo encontrada com grande infestação em Kielmeyera rugosa, porém também ocorreu em Acosmium bijugum e Chamaecrista cytisoides (Dantas \& Ribeiro 2008). Duas epífitas foram encontrada nas Areias Brancas, uma delas foi Aechmea mertensii, espécie amplamente distribuida nos estados das regiões Norte e Nordeste do Brasil e nos paises da América Latina (Martinelli et al. 2008).

Em estudo nas restingas do Nordeste do Estado da Bahia, Pinto et al. (1984) descrevem a fitofisionomia dos "tabuleiros costeiros", nela ocorrem os principais gêneros citados para as Areias Brancas do Parque Nacional Serra de Itabaiana - Poaceae (Axonopus, Paspalum, Trachypogon); Arecaceae (Attalea, Bactris, Syagrus); Polygonaceae (Coccoloba); Fabaceae (Diptychandra, Cassia, Stryphnodendron); Rutaceae (Esenbeckia); Dilleniaceae (Curatella); Apocynaceae (Hancornia, Himatanthus), dentre outros. Das espécies encontradas nas Areias Brancas do Parque Nacional Serra de Itabaiana, $32 \%$ estão citadas na listagem da Flora das Restingas do Nordeste da Bahia (Pinto et al. 1984). Na listagem das espécies de fanerógamas das Restingas de Pernambuco (Zickel et al. 2007) está proporção é de $26 \%$.

Estrutura da vegetação - Na análise fitossociológica das Areias Brancas abertas foram amostrados no estrato arbustivo - arbóreo 99 indivíduos, distribuídos em 19 espécies, num total de 12 famílias. A densidade total estimada foi de 286 ind ha ${ }^{-1}$, cobertura vegetal de $51 \%$, Índice de Diversidade de Shannon-Weinner 55,13 nat e Equabilidade 0,42. Nas Areias Brancas fechadas, foi registrada a ocorrência de 112 indivíduos de 16 espécies em 10 famílias; A densidade foi de 635 ind ha-1, cobertura vegetal total de $75 \%$, índice de diversidade igual a 70,68 nat e Equabilidade 0,46. Os valores de Diversidade foram considerados baixos, avaliando-se os valores de Equabilidade de ambas as áreas, que indicaram que menos de $50 \%$ da diversidade teórica máxima foi encontrado, reflexo da dominância de espécies como Coccoloba laevis, nas Areias Brancas abertas e Agarista revoluta para as Areias Brancas fechadas.

$\mathrm{O}$ quociente de similaridade de Sorensen para a comunidade vegetal das duas áreas foi de 0,63 demonstrando uma alta similaridade entre as duas floras. Das 23 espécies encontradas na amostra, 11 foram comuns às duas áreas, sendo que, as espécies mais abundantes, como $C$. laevis e $A$. revoluta habitavam ambas fitofisionomias. Nenhuma das espécies incomuns às duas fisionomias possuiu abundância maior que quatro, demonstrando que estas espécies não são representativas das fitofisionomias em questão.

A grande diferença de densidade entre as áreas e sua alta semelhança florística levantam a hipótese dessas áreas fazerem parte de estágios sucessionais distintos de uma mesma fitofisionomia. A maior diversidade encontrada na área de Areia Branca fechada também fortalece a hipótese sobre a sucessão. Segundo Wittaker (1972), as comunidades vegetais tendem a aumentar a sua diversidade específica no curso da sucessão.

O estudo da distribuição espacial da vegetação revelou que ambas as áreas estudadas possuem distribuição agregada $\left(\operatorname{IAP}_{1}=3,7 ; \operatorname{IAP}_{2}=2,1\right.$; para áreas abertas e fechadas respectivamente), indicando a tendência de a vegetação ocorrer associada em moitas. A distribuição agregada de uma comunidade pode ser resultado da predisposição social em formar grupos, das distribuições agregadas de recursos e das tendências da prole em permanecerem unidas a seus pais (Ricklefs 1996, Jansen 1982).

As fisionomias das Areias Brancas provavelmente vêm sendo alteradas pelas constantes queimadas e retirada de madeira (Vicente et al. 2005). A similaridade entre as áreas abertas e fechadas, associada aos parâmetros de densidade e diversidade, revela a possibilidade de que ao longo dos processos sucessionais a vegetação adensa e torna-se mais diversa, como sugerido por Fuentes et al. (1986) e Hacker \& Gaines (1997) para vegetações abertas. A agregação de plantas em agrupamentos é uma estratégia de colonização a qual as comunidades das moitas tornam se mais complexas, aumentando sua diversidade e sua cobertura vegetal a partir de uma planta focal facilitadora (Kikvidze \& Nakhutsrishvili 1998, Zaluar \& Scarano 2000). 
A diminuição considerável do índice de agregação encontrada na vegetação de Areias Brancas abertas em relação às Areias Brancas fechadas revela que o adensamento da comunidade tende a diminuir a tendência da associação em moitas, favorecendo uma maior cobertura vegetal à medida que as plantas colonizam as áreas de solo exposto entre as moitas (Zaluar \& Scarano 2000).

Fitossociologia - A importância fitossociológica de Coccoloba laevis (Polygonaceae) nas áreas abertas foi caracterizada pela maior densidade, freqüência e dominância na vegetação local, totalizando um IVI de 1,35 , representando $45 \%$ do total. Acosmiun bijugum (Fabaceae) é a segunda em IVI, $(0,23)$, diferenciando-se pela grande dominância em relação às demais espécies, por se tratarem de indivíduos com grande circunferência de tronco para a área. Nas áreas de Areias Brancas fechadas, Agarista revoluta (Ericaceae) apresentou maior IVI $(1,00)$ e Chamaecrista cytisoides (Fabaceae) a segunda, com IVI igual a 0,4 . Há ocorrência de similaridade florística significativa entre as duas áreas analisadas. Entretanto as áreas apresentam diferença entre as espécies mais frequentes e abundantes (tabela 2).

As plantas amostradas agrupam-se nas classes iniciais de diâmetro de caule, com poucos indivíduos nas classes de maior tamanho, indicando que a comunidade está em crescimento (Martins 1993). Este padrão é esperado para vegetações secundárias, apresentando-se em uma curva gráfica em forma de " $\mathrm{J}$ " invertido. Pode-se afirmar que as fisionomias atuais das Areias Brancas encontram-se em pleno estágio sucessional, dirigindo-se a estágios mais avançados. O padrão em "J" invertido sugere, também, a existência de um contingente de indivíduos jovens que irão suceder àqueles que já se encontram senis (Lopes et al. 2002) ou que está havendo um período de crescimento da comunidade (Jansen 1980). Observou-se diferença significativa dentre o diâmetro do caule das duas áreas amostras (Student $=3,5, \mathrm{GL}=209$, $P<0,05)$. Segundo Mueller-Dombois \& Ellenberg (1974), relacionar diâmetro de caule com o tempo de regeneração da vegetação de cada habitat só é possível para áreas com composições florísticas similares ou com tempo de crescimento de suas plantas semelhante. As áreas abertas e fechadas, por possuírem índice de similaridade de Sorensen significativo $(0,63)$, podem ser comparadas e observa-se que Areias Brancas abertas é a vegetação em estágio mais inicial de regeneração, em relação às Areias Brancas fechadas.

O dossel da vegetação é composto principalmente por arbustos entre $3 \mathrm{~m}$ e $6 \mathrm{~m}$, que possuem caule múltiplo, e raras árvores maiores de sete metros. Arbustos também são comuns no sub-dossel. A altura média da comunidade vegetal é de 4,6 2,1 m (Areias Brancas aberta) e 4,0 $\pm 1,2 \mathrm{~m}$ (Areias Brancas fechada). São vegetações com alturas significativamente diferentes (Student $=2,14, \mathrm{GL}=209, P<0,05$ ). A maior altura relativa da comunidade de vegetação aberta em relação à comunidade de vegetação fechada é contraditória a afirmações que em comunidades com sucessão mais avançada a altura do dossel é maior que as comunidades em sucessão mais inicial (Martins 1993). Esta diferença pode ter explicação nas características de vida das espécies dominantes de ambas as áreas.

A distribuição das freqüências nas classes de altura não descreve o mesmo padrão de "J" invertido como a distribuição das freqüências em classes diamétricas. Nas Areias Brancas abertas o padrão em "J" invertido configura-se a partir da terceira classe de altura. Nas duas iniciais, o critério de inclusão dos indivíduos na amostra, provavelmente exclui aqueles com altura correspondente a estas classes, mas que não haviam atingido CAP de $15 \mathrm{~cm}$ (Lopes et al. 2002), $\mathrm{Na}$ altura de cinco a seis metros, observa-se um pico de freqüência. Incidentes catastróficos como fogo, corte ou doenças em períodos anteriores podem ter ocasionado este acúmulo de indivíduos nesta classe de altura (Mueller-Dombois \& Ellenberg 1974). A distribuição de freqüência nas classes de altura para as Areias Brancas fechadas descreve o padrão de "J" invertido a partir da terceira classe altimétrica, acentuando a diferença com as Areias Brancas abertas e indicando um melhor nível de preservação para esta área.

A vegetação das Areias Brancas do Parque Nacional Serra de Itabaiana foi inicialmente caracterizada como restinga (Governo de Sergipe 1978), onde se tomou por base o substrato arenoso-quartzoso distrófico, visualmente semelhante ao encontrado nas áreas de restinga do estado. Entretanto, estes depósitos arenosos são formações in loco de origem diferente das areias brancas dos tabuleiros da zona costeira propriamente dita (Dantas \& Ribeiro 2010).

A proximidade com as áreas de Restinga e a existência de espécies vegetais comuns não são suficientes para caracterizar a vegetação das Areias Brancas como restinga, tão pouco como cerrado, pois de acordo com Eiten (1983), características climáticas de relevo e origem são fundamentais para a caracterização de um tipo vegetacional.

Em sentido botânico, considera-se "vegetação de restinga" o conjunto de comunidades vegetais fisionomicamente distintas que habitam as areias Quaternárias de deposição flúvio-marinha desde o 
Tabela 2. Parâmetros fitossociológicos das espécies amostradas nos dois habitats das Areias Brancas no Parque Nacional Serra de Itabaiana, Sergipe-Brasil, ordenados decrescentemente pelo IVI. (Abun = Abundância; DA = densidade absoluta (indivíduos ha $\left.^{-1}\right) ; \mathrm{DR}=$ densidade relativa (\%); FA. = freqüência absoluta; FR = freqüência relativa (\%); $\mathrm{AB}=$ área basal absoluta $\left(\mathrm{m}^{2}\right)$; DoA = dominância absoluta $\left(\mathrm{m}^{2} \mathrm{ha}^{-1}\right) ;$ DoR = dominância relativa; IVI = índice de valor de importância).

Table 2. Phtosociological parameters of sampled species in two habitats of Areias Brancas at National Park Serra de Itabaina, Sergipe-Brazil, arranged by decresing IVI. (Abun = Abundance; DA = absolute density (individual ha ${ }^{-1}$ ); DR $=$ relative density $(\%) ; \mathrm{FA}=$ absolute frequency; $\mathrm{FR}=$ relative frequency $(\%) ; \mathrm{AB}=$ absolute basal area $\left(\mathrm{m}^{2}\right) ;$ DoA $=$ absolute dominance $\left(\mathrm{m}^{2} \mathrm{ha}^{-1}\right)$; DoR $=$ relative dominance; $\mathrm{IVI}=$ importance index $).$

\begin{tabular}{|c|c|c|c|c|c|c|c|c|c|}
\hline Espécie & Abun & DA & $\mathrm{DR}$ & FA & FR & $\mathrm{AB}$ & DoA & DoR & IVI \\
\hline \multicolumn{10}{|l|}{ AREIAS BRANCAS ABERTAS } \\
\hline Coccoloba laevis & 54 & 156,00 & 0,55 & 10 & 0,222 & 0,441 & 0,321 & 0,585 & 1,353 \\
\hline Ascomiun bijugum & 4 & 11,56 & 0,04 & 4 & 0,089 & 0,079 & 0,058 & 0,105 & 0,234 \\
\hline Euphorbiaceae sp.1 & 5 & 14,44 & 0,05 & 3 & 0,067 & 0,025 & 0,018 & 0,033 & 0,150 \\
\hline Kielmeyera rugosa & 4 & 11,56 & 0,04 & 4 & 0,089 & 0,012 & 0,009 & 0,016 & 0,145 \\
\hline Diptychandra epunctata & 4 & 11,56 & 0,04 & 4 & 0,089 & 0,010 & 0,007 & 0,014 & 0,143 \\
\hline Manilkara salzmannii & 2 & 5,78 & 0,02 & 2 & 0,044 & 0,054 & 0,039 & 0,072 & 0,136 \\
\hline Hanconia speciosa & 4 & 11,56 & 0,04 & 3 & 0,067 & 0,019 & 0,013 & 0,025 & 0,132 \\
\hline Schefflera vinosa & 4 & 11,56 & 0,04 & 3 & 0,067 & 0,014 & 0,010 & 0,018 & 0,125 \\
\hline Agarista revoluta & 5 & 14,44 & 0,05 & 1 & 0,022 & 0,026 & 0,019 & 0,035 & 0,107 \\
\hline Ouratea cuspidata & 3 & 8,67 & 0,03 & 2 & 0,044 & 0,010 & 0,008 & 0,014 & 0,089 \\
\hline Esenbeckia grandiflora & 1 & 2,89 & 0,01 & 1 & 0,022 & 0,016 & 0,012 & 0,021 & 0,054 \\
\hline Davilla flexuosa & 2 & 5,78 & 0,02 & 1 & 0,022 & 0,008 & 0,006 & 0,011 & 0,053 \\
\hline Stryphnodendron pulcherrimum & 1 & 2,89 & 0,01 & 1 & 0,022 & 0,015 & 0,011 & 0,020 & 0,053 \\
\hline Pera glabrata & 1 & 2,89 & 0,01 & 1 & 0,022 & 0,009 & 0,007 & 0,012 & 0,045 \\
\hline Coccoloba rosea & 1 & 2,89 & 0,01 & 1 & 0,022 & 0,004 & 0,003 & 0,006 & 0,038 \\
\hline Chamaecrista cytisoides & 1 & 2,89 & 0,01 & 1 & 0,022 & 0,004 & 0,003 & 0,005 & 0,037 \\
\hline Guettarda platypoda & 1 & 2,89 & 0,01 & 1 & 0,022 & 0,002 & 0,002 & 0,003 & 0,035 \\
\hline Myrcia guianensis & 1 & 2,89 & 0,01 & 1 & 0,022 & 0,002 & 0,002 & 0,003 & 0,035 \\
\hline \multirow[t]{2}{*}{ Bauhinia acuruana } & 1 & 2,89 & 0,01 & 1 & 0,022 & 0,002 & 0,001 & 0,002 & 0,035 \\
\hline & 99 & 286,00 & 1,00 & 45 & 1,000 & 0,753 & 0,548 & 1,000 & 3,000 \\
\hline \multicolumn{10}{|l|}{ AREIAS BRANCAS FECHADAS } \\
\hline Agarista revoluta & 34 & 192,77 & 0,30 & 8 & 0,170 & 0,696 & 0,546 & 0,525 & 1,00 \\
\hline Coccoloba laevis & 18 & 102,05 & 0,16 & 5 & 0,106 & 0,173 & 0,136 & 0,131 & 0,40 \\
\hline Chamaecrista cytisoides & 21 & 119,06 & 0,19 & 5 & 0,106 & 0,019 & 0,015 & 0,014 & 0,31 \\
\hline Kielmeyera rugosa & 7 & 39,69 & 0,06 & 4 & 0,085 & 0,088 & 0,069 & 0,066 & 0,21 \\
\hline Guettarda platypoda & 6 & 34,02 & 0,05 & 5 & 0,106 & 0,053 & 0,041 & 0,040 & 0,20 \\
\hline Coccoloba rosea & 6 & 34,02 & 0,05 & 3 & 0,064 & 0,040 & 0,031 & 0,030 & 0,15 \\
\hline Guettarda angelica & 4 & 22,68 & 0,04 & 3 & 0,064 & 0,026 & 0,020 & 0,019 & 0,12 \\
\hline Euphorbiaceae sp.1 & 3 & 17,01 & 0,03 & 2 & 0,043 & 0,042 & 0,033 & 0,031 & 0,10 \\
\hline Jacaranda obovata & 3 & 17,01 & 0,03 & 3 & 0,064 & 0,010 & 0,008 & 0,008 & 0,10 \\
\hline Humiria balsamifera & 3 & 17,01 & 0,03 & 2 & 0,043 & 0,033 & 0,026 & 0,025 & 0,09 \\
\hline Clusia sp. & 1 & 5,67 & 0,01 & 1 & 0,021 & 0,080 & 0,062 & 0,060 & 0,09 \\
\hline Hanconia speciosa & 2 & 11,34 & 0,02 & 2 & 0,043 & 0,006 & 0,005 & 0,005 & 0,07 \\
\hline Acosmiun bijugum & 1 & 5,67 & 0,01 & 1 & 0,021 & 0,045 & 0,035 & 0,034 & 0,06 \\
\hline Myrcia fallax & 1 & 5,67 & 0,01 & 1 & 0,021 & 0,010 & 0,008 & 0,007 & 0,04 \\
\hline Myrcia guianensis & 1 & 5,67 & 0,01 & 1 & 0,021 & 0,004 & 0,003 & 0,003 & 0,03 \\
\hline \multirow[t]{2}{*}{ Pera glabrata } & 1 & 5,67 & 0,01 & 1 & 0,021 & 0,002 & 0,002 & 0,002 & 0,03 \\
\hline & 112 & 635,00 & 1,00 & 47 & 1,000 & 1,326 & 1,039 & 1,000 & 3,000 \\
\hline
\end{tabular}


Pleistoceno (Rizzini 1979). Caracterizar as Areias Brancas como Restingas seria modificar o conceito para que possa incluir vegetações em regiões sem influência da deposição marinha, como no caso das Areias Brancas do Parque Nacional Serra de Itabaiana, onde não é caracterizada tal influência, pois nenhuma das incursões marítimas, formadoras de depósitos de sedimento arenoso na costa de Sergipe, afetou esta região (Bittencourt et al. 1983).

As Areias Brancas do Parque Nacional Serra de Itabaiana devem ser consideradas como um Refúgio Ecológico, pelo sistema de Classificação do IBGE (1992), uma vegetação azonal derivada de condições especiais de formação do substrato. Alves et al. (2007) referemse a este tipo de vegetação azonal como associada aos campos rupestres, ocorrendo em áreas tabulares de deposição sedimentar, em áreas mais elevadas que os tipos vegetacionais do entorno. A similaridade florística das Areias Brancas do Parque Nacional Serra de Itabaiana pode ser explicada pela hipótese levantada por Alves et al. (2007) sobre a existência de diferentes vegetações associadas a afloramentos rochosos agindo como "trampolim" de espécies rupestres do cerrado e da caatinga para a restinga.

As Areias Brancas do Parque Nacional Serra de Itabaiana estão sob forte influência florística das demais vegetações abertas próximas, como a Caatinga e a Restinga, do mesmo modo que as Areias Brancas amazônicas sofrem influência do Cerrado e da própria Caatinga (Macedo 1977, Macedo \& Prance 1978, Anderson 1981).

Entre as duas fitofisionomias de Areias Brancas avaliadas neste estudo, ficaram claras suas relações de semelhança florístico-estrutural, indicando que estas não devem ser consideradas como vegetações distintas e sim como estágios sucessionais diferentes de uma mesma formação vegetal. As diferenças encontradas podem ser consideradas como mecanismos da dinâmica sucessional da vegetação.

Agradecimentos - Ao Deutscher Akademischer Austhausc Dienst (DAAD) pelo financiamento (processo A/06/00534); aos estagiários do Herbário ASE; ao Ibama, pela logística de campo, e ao professor Dr. Celso Morato, pelas correções preliminares e discussão de idéias.

\section{Referências bibliográficas}

ALVES, R.J.V., CARDIN, L. \& KROFP, M.S. 2007. Angiosperm disjunction "Campos Rupestres Restingas" - a re-evaluation. Acta Botanica Brasilica 21:675-685.

ANDERSON, A.B. 1981. White sand vegetation of Brazilian Amazonia. Biotropica 13:199-210.
ANDRADE, J.A. \& SANTOS, A.F. 1985. A cobertura vegetal de Sergipe. In Anais da I Reunião da Sociedade Brasileira para o Progresso da Ciência - SBPC, Regional Nordeste. João Pessoa, v.1, p.5-13.

APG III. 2009. An update of the Angiosperm Phylogeny Group classification for the orders and families of flowering plants: APG III. Botanical Journal of the Linnean Society 161:105-121.

BITTENCOURT, A.C.S.P., MARTIN, L., DOMINGUEZ, J.M.L. \& FERREIRA, Y.A. 1983. Evolução paleogeográfica quaternária da costa do estado de Sergipe e da costa sul do estado de Alagoas. Revista Brasileira de Geociências 13:93-97.

BOGHOSSIAN, M.R., PEIXOTO, P.V., BRITO, M.F. \& TOKARNIA, C.H. 2007. Aspectos clínico-patológicos da intoxicação experimental pelas sementes de Crotalaria mucronata (Fabaceae) em bovinos. Pesquisa Veterinaria Brasileira 27:149-156.

BRITTO, I.C., QUEIROZ, L.P., GUEDES, M.L.S., OLIVEIRA, N.C. \& SILVA, L.B. 1993. Flora fanerogâmica das dunas e lagoas de Abaeté, Salvador, Bahia. Sitientibus 11:31-46.

BROWER, J.E., ZAR, J.H. \& VON ENDE, C.N. 1997. Field \& laboratory methods for general ecology. $4^{a}$ ed. W.C. Brown Publishers, Iowa.

CATANA, J.R. \& ANTHONY, J. 1963. The wandering quarter method of estimating population density. Ecology 44:349-360.

CUATRECASAS, J.A. 1961. A taxonomic revision of Humiriaceae, Contribuitions from the United States National Herbarium. Bulletin of the United States National Museum 35:25-214.

DANTAS, T.V.D. \& RIBEIRO, A.S. 2008. Infestação da erva-de-passarinho (Psittacanthus dichrous Mart. ex. Schult (Loranthaceae) em Kielmeyera rugosa Choisy (Clusiaceae) no Parque Nacional Serra de Itabaiana, Sergipe. Biologia Geral e Experimental 8:7-14.

DANTAS, T.V.P. \& RIBEIRO, A.S. 2010. Estrutura populacional de Kielmeyera rugosa Choisy (Clusiaceae) no Parque Nacional Serra de Itabaiana, Estado de Sergipe. Acta Scientiarum Biological Sciences 32:141-146.

DAVID, J.M., CRUZ, F.G., GUEDES, M.L.S. \& CHÁVEZ, J.P. 1996. Flavonol glycosides from Davilla flexuosa. Journal of Brazilian Chemistry Society 7:115-118.

EITEN, G. 1983. Classificação da vegetação do Brasil. Conselho Nacional de Desenvolvimento Científico e Tecnológico, Brasília.

EMBRAPA. 1975. Levantamento exploratório: reconhecimento de solos do estado de Sergipe. Boletim técnico. Embrapa/Sudene, Recife.

FIDALGO, O. \& BONONI, V.L. 1984. Guia de coleta, preservação e herborização de material botânico. Manual no 4. Instituto de Botânica, São Paulo.

FRANCO, E. 1983. Biogeografia de Sergipe. Segrase, Governo de Sergipe, Aracaju. 
FUENTES, E.R., HOFFMANN, A.J., POIANI, A. \& ALLIENDE, M.C. 1986. Vegetation change in large clearings: patterns in the Chilean matorral. Oecologia 68:358-366.

GONÇALVES, L.M.C. \& ORLANDI, R.P. 1983. Vegetação In Projeto RadamBrasil, folhas SC 24/25 Aracaju/ Recife. Levantamento de Recursos Naturais 30, Rio de Janeiro, p.573-639.

GOVERNO DE SERGIPE. 1976. Zoneamento ecológico florestal do estado de Sergipe. Governo de Sergipe, Aracaju.

GOVERNO DE SERGIPE. 1978. Projeto básico para a implantação da Estação Ecológica Serra de ItabaianaSE. Governo de Sergipe, Aracaju.

HACKER, S.D. \& GAINES, S.D. 1997. Some implications of direct positive interactions for community species diversity. Ecology 78:1990-2003.

HOLMGREN, P.K. \& HOLMGREN, N.H. 1998. Index Herbariorum: A global directory of public herbaria and associated staff. New York Botanical Garden's Virtual Herbarium. Nova Iorque. [continuamente atualizado] http://sweetgum.nybg.org/ih/ (acesso em 05/01/2008).

IBGE. 1992. Manual técnico da vegetação brasileira. Série de manuais técnicos em geociências 1. IBGE, Rio de Janeiro.

IBGE. 2004. Mapa de vegetação do Brasil. Diretoria de geociências. 3aa ed. IBGE, Brasília.

JANZEN, D.H. 1982. Differential seed survival and passage rates in cows and horses, surrogate Pleistocene dispersal agents. Oikos 38:150-156.

KIKVIDZE, Z. \& NAKHUTSRISHVILI. 1998. Facilitation in subnival vegetation patches. Journal of Vegetation Science 9:261-264.

KÖPPEN, W. \& GEIGER, R. 1928. Klimate der Erde. Verlag Justus Perthes, Gotha.

LOPES, W.P., SILVA, A.F., SOUZA, A.L. \& MEIRA NETO, J.A.A. 2002. Estrutura fitossociológica de um trecho de vegetação arbórea no Parque Estadual do Rio Doce-Minas Gerais, Brasil. Acta Botanica Brasilica 16:443-456.

MACEDO, M. 1977. Dispersão de plantas lenhosas de uma campina Amazônica. Acta Amazonica 7:1-69.

MACEDO, M. \& PRANCE, G.T. 1978. Notes the vegetation of Amazonia II. The dispersal of plants in Amazoniam white sand Campinas; the campinas as functional Islands. Brittonia, 30:203-215.

MARTINELLI, G., VIEIRA, C.M., GONZALEZ, M., LEITMAN, P., PIRATININGA, A., COSTA, A.F., FORZZA, R.C. 2008. Bromeliáceas da Mata Atlântica brasileira: lista de espécies, distribuição e conservação. Rodriguésia 59:209-258.

MARTINS, F.R. 1993. Estrutura de uma floresta mesófila. 2a ed. Universidade Estadual de Campinas - Unicamp, São Paulo.

MUELLER-DOMBOIS, D. \& ELLENBERG, H. 1974. Aims and methods of vegetation ecology. Willey \& Sons. New York.
PAYANDEH, B. 1970. Comparison of method for assessing spacial distribution of trees. Forest Science 16:312-317.

PEREIRA, M.S. \& ALVES, R.R.N. 2007. Composição florística de um remanescente de Mata Atlântica na Área de Proteção Ambiental Barra do Rio Mamanguape, Paraíba, Brasil. Revista de Biologia e Ciências da Terra 7:1-10.

PEREIRA, M.S. \& BARBOSA, M.R.V. 2004. A família Rubiaceae na Reserva Biológica Guaribas, Paraíba, Brasil. Subfamílias Antirheoideae, Cinchonoideae e Ixoroideae. Acta Botanica Brasilica 18:305-318.

PIELOU, E.C. 1975. Ecological diversity. Wiley \& Sons, New York.

PILGER, R. 1927. Plantae Luetzelburgianae brasilienses VII. Notizblatt des Königl. botanischen Gartens und Museums zu Berlin 91:43-54.

PINTO, G.C.P., BAUTISTA, H.P. \& FERREIRA, J.D.C.A. 1984. A restinga do litoral nordeste do estado da Bahia. In Restingas: origem, estrutura, processos (L.D. Lacerda, D.S.D. Araujo, R. Cerqueira \& B. Turcq, orgs.). Ceuff, Niterói, p.195-216.

QUEIROZ, E.P. 2007. Levantamento florístico e georreferenciamento das espécies com potencial econômico e ecológico em restinga de Mata de São João, Bahia, Brasil. Biotemas 20:41-47.

RICKLEFS, R.E. 1996. A economia da natureza. 3a ed. Guanabara-Koogan, Rio de Janeiro.

RIZZINI, C.T. 1979. Tratado de fitogeografia do Brasil. vol. 2. Hucitec/Edusp, São Paulo.

RODAL,M.J.R.\&NASCIMENTO,L.M.2002. Levantamento florístico da floresta serrana da Reserva Biológica de Serra Negra, microrregião de Itaparica, Pernambuco, Brasil. Acta Botanica Brasilica 16:481-500.

SADDI, N. 1994. Phytogeographical study in the genus Kielmeyera Martius (Guttiferae). Publicações Avulsas do Herbário Central, Cuiabá.

VIANA, B.F., SILVA, F.O. \& KLEINERT, A.M.P. 2006. A flora apícola de uma área restrita de dunas litorâneas, Abaeté, Salvador, Bahia. Revista Brasileira de Botânica 29:13-25.

VICENTE, A., DE ARAÚJO, G.M.M., LÍRIO, G.P. \& SANTOS, S.C. 1997. Descrição parcial e preliminar dos hábitats da Serra de Itabaiana, Sergipe. Publicações Avulsas do Centro Acadêmico Livre de Biologia da Universidade Federal de Sergipe 1:23-27.

VICENTE, A., RIBEIRO, A.S., SANTOS, E.A. \& FRANCO, C.R.P. 2005. Levantamento botânico In: Parque Nacional Serra de Itabaiana - Levantamento da Biota (C.M. Carvalho \& J.C. Vilar, coord.). Ibama, Biologia Geral e Experimental/UFS, Aracaju, p.15-37.

VICENTE, A. 1999. Levantamento florístico de um fragmento florestal na Serra de Itabaiana - Sergipe. Dissertação de mestrado, Universidade Federal Rural de Pernambuco, Recife. 
VILAR, J.C., ZYNGIER, N.A.C. \& CARVALHO, C.M. 2000. Distribuição espacial de Vellozia dasypus Seubert (Veloziaceae) e Melocactus zehntneri (Britt. et Rose) Lutzelb. (Cactaceae) na Serra de Itabaiana, Sergipe. Biologia Geral e Experimental 1:5-15.

WITTAKER, R.H. 1972. Evolution and measurements of species diversity. Taxon 21:213-251.
ZALUAR, H.L.T. \& SCARANO, F.H. 2000. Facilitação em restingas de moitas: um século de buscas por espécies focais. In: Ecologia de restingas e lagoas costeiras. (F.A. Esteves \& L.D. Lacerda, eds.) Nupem/Universidade Federal do Rio de Janeiro - UFRJ, Macaé.

ZICKEL, C.S., ALMEIDA-JR, E.B., MEDEIROS, D.P.W., LIMA, P.B., SOUZA, T.M.S. \& LIMA, A.B. 2007. Magnoliophyta species of restinga, state of Pernambuco, Brazil. Check List 3:224-241. 